\title{
Performance Evaluation of DWDM for Radio over Fiber System with Dispersion Compensation and EDFA
}

\author{
Husam Abduldaem Mohammed \\ Instructor \Baghdad University $\backslash$ College of Engineering $\backslash$ Electronic and Communication Engineering \\ Department.
}

\begin{abstract}
The radio-over-fiber (RoF) system is one of the potential schemes for the future broadband wireless communication systems such as mobile communications, hotspots and suburban areas. The use of Dense Wavelength Division Multiplexing WDM system is responding to the demands for high data rate applications and reasonable mobility for broadband communication.
\end{abstract}

The work aims to investigate the performance of DWDM system utilizing Eribium Dopped Fiber amplifiers EDFAs and Dispersion Compensating Fiber DCF for different length of optical fiber and bit rates. The most effective factors causes performance degradations are the attenuation and dispersion. EDFA was introduced in the proposed system model as a solution to encounter the effects of attenuation and scattering losses, while the DCF utilized to mitigate the effect of dispersion.

The simulation was implemented using optiwsystem 7 and matlab R2011a. The results show that the use of EDFA and DCF make significantly boosts the performance of DWDM RoF system and increases the length of the fiber at which a Bit Error Rate BER of $10^{-9}$ can be obtained.

Keywords: DWDM, EDFA, DCF, BER, Q-factor

\section{INTRODUCTION}

Wavelength division multiplexing (WDM) is a technology which multiplexes multiple optical carrier signals on a single optical fiber by using different wavelengths of laser light to carry different signals. This allows for a multiplication in capacity of an optical fiber by adding new channels, each channel on a new wavelength of light, in addition to enabling bidirectional communications over one strand of fiber. [1]. The term wavelength-division multiplexing is commonly applied to an optical carrier (which is typically described by its wavelength), whereas frequency-division multiplexing typically applies to a radio carrier (which is more often described by frequency). Since wavelength and frequency are tied together through a simple directly inverse relationship, the two terms actually describe the same concept [2].

Wavelength-division multiplexing (WDM) gives better utilization of the large bandwidth of optical fiber and can increase the capacity of the cable network. Through WDM, signals from two or more line systems are transmitted over the same fiber [3]. The signal from different sources which combined by a multiplexer and fed into an optical fiber, channels combined are separated in the receiver unit by a demultiplexer and detected by photodetector [3]. In WDM, each electrical data channel $\mathrm{i}$ is modulated onto its optical carrier wave with a wavelength $\lambda_{\mathrm{i}}$. Therefore one subdivides the optical transmission band $\mathrm{B}_{\text {opt }} \sim 20 \mathrm{THz}$, resp. $\Delta \lambda_{\text {opt }} \sim 200 \mathrm{~nm}$, into $\mathrm{N}(\sim 100-1000)$ bands $\left(\mathrm{B}=\mathrm{B}_{\mathrm{opt}} / \mathrm{N}\right)$. To extract a particular channel at the receiver, the demultiplexer has to filter one particular channel at the desired wavelength $\lambda_{\mathrm{i}}$ by a narrow band optical filter $\left(\Delta \mathrm{f}_{\text {filter }}\right.$ $\sim \mathrm{B})$ and direct it to the photodetector [4]. The optical multiplexing operation of different data channels occurs in the frequency domain into nonoverlapping frequency bands $\mathrm{B}$ (because $\mathrm{N}$ transmission wavelengths $\lambda_{1}-\lambda_{N}$ exist which are unrelated in time) [4]. This technique can be implemented to not only design ultra high capacity optical communication systems, but also upgrade the existing systems. WDM takes advantage of the almost infant amount of the fiber bandwidth and the availability of fiber amplifiers in the 1530-1560 nm wavelength windows [3]. The WDM devices at the transmitting unit is essentially a power combining referred to as a multiplexer. The device at the receiver unit is called a demultiplexer and should ideally separate out various channels with negligible loss and signal distortion. a large number of channels can be combined and separated with angularly dispersive multiplexing elements. At the output of the multiplexer, these light rays become co-linear and can be easily launched simultaneously into an optical fiber. A WDM works in exactly the reverse fashion, directing light beams of various wavelengths from a fiber into their respective channels [3].

Multiplexers and demultiplexers can be divided into either passive or active in design. Passive designs are based on prisms, gratings or filters, while active designs id a combination of passive devices with tunable filters [1].The primary challenges in these devices is to minimize cross-talk and maximaze channel separation. Cross talk is a measure of how well the channels are separated, while channel separation refers to the ability to distinguish each wavelength. 
The use of WDM for the distribution of RoF signals has gained importance. WDM enables the efficient exploitation of the fiber network's bandwidth. Its application in RoF networks has many advantages including simplification of the network topology by allocating different wavelengths to individual BSs, enabling easier network and service upgrades and providing simpler network management [1].

If the $\mathrm{N}$ transmitters in a DWDM link such as shown in Figure (1), operate at bit rates of $B_{1}$ through $B_{N}$, respectively, then the total bandwidth is $[5,6]$

$$
B=\sum_{i=1} B_{i}
$$

When all the bit rates are equal, then the system capacity is enhanced by a factor $\mathrm{N}$ as compared with a single-channel link.

In fiber optic communication systems, the error rates usually range from $10^{-9}$ to $10^{-12}$. This value depends on the SNR the receiver. The BER can be calculated from the Q-Factor as shown in [5, 6]

$$
\begin{aligned}
& B E R=\frac{1}{2} \operatorname{erfc}\left(\frac{Q}{\sqrt{2}}\right)=\frac{\exp \left(-Q^{2} / 2\right)}{\sqrt{2 \pi} Q} \\
& Q=\frac{I_{1}-I_{o}}{\sqrt{\sigma_{1}^{2}}+\sqrt{\sigma_{o}^{2}}}
\end{aligned}
$$

$I_{1}$ and $I_{0}$ are the average values when the bits transmitted are 1 and 0 respectively in the bit stream. $\sigma_{1}^{2}$ and $\sigma_{0}^{2}$ are corresponding variances when 1 and 0 are received. Critical requirements of a WDM-system are the generation of the extremely stable carrier frequency $\omega_{\mathrm{i}}$ with $\Delta \omega_{\mathrm{i}}<<\mathrm{B}$ and the precise and narrowband wavelength filtering, as well as the system complexity and the high number of optical components for large channel numbers.

The main advantages of using WDM system are the relative low data rate per optical channel with modest requirements for dispersion and electronic speed and data format and timing of the channels are independent of each other. While the main drawbacks of the system are the requirements of highly stable optical frequency generation and small oscillator bandwidth $(\sim \mathrm{MHz})$, also, high total operational power $\left(\mathrm{N}^{*} \mathrm{P}_{\text {op.channel }}\right.$; typically $200 * 1 \mathrm{~mW} / \mathrm{ch}=200 \mathrm{~mW}$ ) in the fiber can result in optical nonlinearities.

The narrow channel spacing in dense WDM link gives rise to crosstalk, which is defined as the feed through of one channel's signal into another channel. Crosstalk can be introduced by almost any component in a WDM system, including optical filters, wavelength multiplexers, optical switches, optical amplifiers, and the fiber itself $[5,6]$.
The EDFA is a length of glass fiber that has doped with the rare-earth metal Erbium ions. These ions act as an active medium with the potential to experience inversion of carriers and emit spontaneous and stimulated emission light near a desirable signal wavelength. The pump is typically another light source whose wavelength is preferentially absorbed by the ions, 0.98 or $1.48 \mathrm{~mm}$ for EDFA. The pump and signal $(1.55 \mathrm{~mm})$ must combined, typically by a wavelength-selective coupler (WDM), and may co- or counter-propagate with respect to each other inside the doped length of fiber. Therefore, the light is absorbed by the doped fiber at a certain pump wavelength and then produces gain for a signal at a different wavelength. Since the transmission and the active medium are both fiber based, the insertion losses are minimal. The gain exhibits a peak at $1530 \mathrm{~nm}$ and a relatively flat region near $1555 \mathrm{~nm}[5]$.

\section{DISPERSION COMPENSATION}

The main goal in any communication system is to increase the transmission distance. Loss and dispersion are the main factor that cause signal degradations and affect fiber-optical communication being the high-capacity develops. The EDFA is the gigantic change happened in the fiber-optical communication system; the loss is no longer the major factor to restrict the fiber optical transmission. Since EDFA works in $1550 \mathrm{~nm}$ wave band, the average single mode fiber (SMF) dispersion value in that wave band is very big, about $15-20 \mathrm{ps} /$ (nm.km1) [8]. It is easy to see that the dispersion become the major factor that restricts long distance fiberoptical transfers as the bit rate increases $[9,10]$.

Dispersion causes the time slot and profile of an optical pulse to change in the course of propagation, causing intersymbol interference ISI and hence causes bit errors in reception. Dispersion is typically measured as a time spread per distance traveled $(\mathrm{s} / \mathrm{km})$. In single-mode fiber performance is primarily limited by chromatic dispersion (also called group velocity dispersion), which occurs because the index of the glass varies slightly depending on the wavelength of the light, and light from real optical transmitters necessarily has nonzero spectral width (due to modulation) $[11,12]$. Polarization mode dispersion, another source of limitation, occurs because although the single-mode fiber can sustain only one transverse mode, it can carry this mode with two different polarizations, and slight imperfections or distortions in a fiber can alter the propagation velocities for the two polarizations. This phenomenon is called birefringence. Mode birefringence $\mathrm{Bm}$ is defmed as the follow Formula [10]:

$B_{m}=\frac{\left|\beta_{x}-\beta_{y}\right|}{k_{o}}=n_{x}-n_{y}$

$\mathrm{n}_{\mathrm{x}}, \mathrm{n}_{\mathrm{y}}$ are the effective refractive of the two orthogonal polarizations. For a given $\mathrm{B}_{\mathrm{m}}$, its fast axis and slow axis components will be formed the phase difference after the light waves transmission 
$\mathrm{L} \mathrm{Km}$. Degree of pulse broadening can be expressed by different group delay $\Delta \tau[10]$.

$$
\begin{aligned}
& \Delta \tau=\left|\frac{L}{v_{g x}}-\frac{L}{v_{g y}}\right|=\frac{d}{d \omega}\left(\beta_{x}-\beta_{y}\right) L= \\
& \left(\frac{n_{x}-n_{y}}{c}+\frac{\omega d\left(n_{x}-n\right)_{y}}{c d \omega}\right) L
\end{aligned}
$$

Dispersion increased the pulse shape distortion caused by the self-phase modulation dispersion (SPM); the other hand, dispersion in WDM systems can also increase the cross-phase modulation, fourwave mixing ( FWM) and other nonlinear effects[10, 13, 14].

several dispersion compensation technologies were proposed[10, 15]. Amongst the various techniques proposed in the literature, the ones that appear to hold immediate promise for dispersion compensation and management could be broadly classified as: dispersion compensating fibers (DCF), chirped fiber Bragg gratings (FBG), and high-order mode (HOM) fiber [16].

There is positive second-order and third-order dispersion value in SMF (single mode fiber), while the DCF dispersion value is negative. So, by inserting a DCF, the average dispersion is close to zero [17]. As the local dispersion of higher transmission link, FWM and XPM were ignored; only to consider the role of SPM and dispersion, the signal transmission can be simulate by solve the nonlinear Schrodinger equation.

In the formula, $\mu_{p}$ is the dispersion compensation rate of p-channel.

$$
\mu_{p}=\left|\frac{D_{D C F}\left(\lambda_{P}\right) L_{D C F}}{D_{S M F}\left(\lambda_{P}\right) L_{S M F}}\right|
$$

$\mathrm{L}_{\mathrm{SMF}}$ and $\mathrm{L}_{\mathrm{DCF}}$ are the conventional single-mode fiber length and dispersion compensation fiber length within the amplifier spacing. $\Delta \lambda$ is the channel wavelength spacing. $\mathrm{D}_{\mathrm{DCF}}\left(\lambda_{\mathrm{p}}\right)$ and $\mathrm{D}_{\mathrm{SMF}}\left(\lambda_{\mathrm{p}}\right)$ are the dispersion coefficient of conventional single mode fiber and dispersion compensation fiber at the $\lambda_{\mathrm{p}}$ wavelength [10].

\section{SYSTEM SIMULATION AND RESULTS}

The work aimed to study the performance of an eight channel DWDM system including the use of single mode fiber SMF, EDFA and DCF. The proposed system is shown in Figure (2). This system uses only SMF and consists of eight channels to be multiplexed using DWDM system. In each there is a digital data generates by Pseudo Random Bit Sequence (PRBS) which will be modulated using MZM modulator with varies number of subcarrier which was in gigahertz. The center frequency range of laser is 194.1-194.8 THZ each with power of 0 $\mathrm{dBm}$. The NRZ (Non-Return to Zero) format was used for signal coding and the modulation used was on-off keying (OOK). These eight signals were multiplexed by the optical multiplexer to be launched into a single SMF. The optical multiplexer has $20 \mathrm{GHz}$ channel bandwidth and $100 \mathrm{GHz}$ channel frequency spacing is used to reduce the crosstalk between adjacent channels. The system was designed so that to minimize noise from the adjacent channels. At the receiver side, these signals were separated by the demultiplexer and each signal passed to a receiver that consists of PIN pdotodiode, filter and a bit error rate BER analyzer.

\subsection{Effect of signal degradation and use of EDFA.}

First the effect of attenuation and dispersion against distance of transmission on the transmitted signal was studied for $1 \mathrm{Gbps}, 2.5 \mathrm{Gbps}$ and $10 \mathrm{Gbps}$ bit rate for each channel. Figures (3) illustrates the Q factor and BER variations with distance of the system. The attenuation coefficient of the standard fiber was considered to be $0.2 \mathrm{~dB} / \mathrm{km}$. The dispersion parameter of $17 \mathrm{ps} / \mathrm{nm} . \mathrm{km}$ and dispersion slop of $0,075 \mathrm{ps} / \mathrm{nm}^{2} . \mathrm{km}$ was considered in the work.

The major influence of dispersion and attenuation signal is limiting the fiber length in order to achieve BER $<10^{-9}$.

\subsection{Effect of EDFA and DCF}

To deal with the degradation effects, one can use the EDFA amplifiers to reduce or cancelling the effect of attenuation. The dispersion compensation techniques can be used to mitigate the distortion comes from dispersion. The dispersion compensation system in the WDM is studied in this paper. Based on optical transmission equation, considering the various types of nonlinear effects and the impact of EDFA,

The use of EDFA, to amplify the incoming optical signal, increases the power of the signal for the same length as in the system block diagram shown in Figure (4). Figure (5) shows how the power varied with distance for $1 \mathrm{Gbps}, 2.5 \mathrm{Gbps}$ and 10 Gbps rates of transmission for SMF only and for SMF with EDFA systems. The EDFA used in this test has a gain of $20 \mathrm{~dB}$ and noise Figure of $4 \mathrm{~dB}$. From the figures, it can be noticed that as the link length increased the power of the arrived signal reduced. For $10 \mathrm{Gbps}$, the degradation effects are more than the other bit rates 1 and 2.5 Gbps.

Figure (6) shows the $Q$ factor and BER variations with distance of the system. It can be noticed that there is an improvement in them as compared to that results uses only SMF in Figures (3).

In the system shown in Figure (7), dispersion compensation is achieved with DCF using postcompensation. Post -compensation scheme achieve dispersion compensation by place the DCF after a certain conventional single-mode fiber.. EDFA is used to compensate the power loss generating by SMF and the DCF signal. Three EDFAs are used in the system as a power amplifier, inline amplifier and preamplifier. The parameters of the DCF fiber used in the simulation includes the dispersion of -85 
$\mathrm{ps} / \mathrm{nm} . \mathrm{km}$ and slope of $-0.3 \mathrm{ps} / \mathrm{nm}^{2} \cdot \mathrm{km}$. the length of the DCF is $10 \mathrm{~km}$ with $0.2 \mathrm{~dB} / \mathrm{Km}$ attenuation coefficient. Figure (8) show the change of $\mathrm{Q}$ factor and BER with distance for $1 \mathrm{Gbps}, 2.5 \mathrm{Gbps}$ and 10 Gbps.

For example the dispersion at a bit rate of $10 \mathrm{Gbps}$ and at a distance of $45 \mathrm{~km}$, where a BER of $10^{-9}$ was obtained for the system uses the SMF only, was 7.19452E6, 8.64253E5 and -3.9095E5 ps/nm for a frequency channel of 193.1, 1.93.2 THz $1.93 .4 \mathrm{THz}$ respectively . But, with the use of $10 \mathrm{~km} \mathrm{DCF}$, the dispersion became 7.18082E6, 5.33279E5 and 1.30018E6 ps/nm for frequency channel of 193.1, 1.93.2 THz 1.93 .4 at $112 \mathrm{~km}$ where a BER of $10^{-9}$ was obtained.

Table 1(1) shows simple comparison for the distances at which the bit error rate equals $10^{-9}$ for 1 , 2.5 and 10 Gbps for SMF only, SMF with EDFA and SMF with EDFA and DCF.

Table (1) shows simple comparison for the distances at which the bit error rate equals $10^{-9}$ for 1, 2.5 and $10 \mathrm{Gbps}$

\begin{tabular}{|l|l|l|l|}
\hline $\begin{array}{l}\text { Bitrate/ } \\
\text { Gbps }\end{array}$ & $\begin{array}{l}\text { SMF } \\
\text { ONLY }\end{array}$ & $\begin{array}{l}\text { SMF+ } \\
\text { EDFA }\end{array}$ & $\begin{array}{l}\text { SMF+EDFA } \\
\text { +DCF }\end{array}$ \\
\hline 1 & 112 & 206 & 265 \\
\hline 2.5 & 100 & 190 & 240 \\
\hline 10 & 45 & 60 & 115 \\
\hline
\end{tabular}

Figures $(9,10$ and 11) shows the eye diagram for the eight users DWDM system for SMF only, SMF and for SMF with EDFA and DCF for bit rates of 1, 2.5 and 10 Gbps respectively. The length shown in the figures are those at which a bit error rates of $10^{-9}$ had been obtained with the systems that uses only SMF.

The spectrum of the optical signal is shown in the Figure (12) for different points in the system for $10 \mathrm{Gbps}$ bit rate.

It shows the spectrum of the modulated laser diode signal, the spectrum of the 8-channel multiplexed signal at output of multiplexer, at the end of SMF, DCF, EDFA, after separated by the demultiplexer and after passing the optical filter.

\section{CONCLUSION}

The effect of degradation factors (attenuation and dispersion) greatly limits the span of the SMF fiber. The use of EDFA greatly compensate the effects of attenuation and scattering losses in the system which makes an improvement on the system performance including the Q factor and BER and increases the length of the link.

The DCF is one technique used as a post compensation to reduce the effect of chromatic dispersion in the SMF and this even assists in increasing the operating bit rate and increased the length of the fiber communication link by reducing the inter symbol interference ISI. It improves the system performance greatly. For example, for 10 Gbps, when the SMF used only the BER of $10^{-9}$ was obtained at a distance of $45 \mathrm{~km}$. When the EDFA added to the system the length extended to $60 \mathrm{~km}$. By using the $10 \mathrm{~km}$ of DCF with SMF and EDFA the BER of $10^{-9}$ was obtained at $115 \mathrm{~km}$ which three time that obtained with the use of SMF only.

\section{REFERENCES}

[1] Sandeep Singh, Neeraj Gupta, Ravi Prakash Shukla and Anamika Sharma, " Simulation of full duplex data transmission in ROF system using Optisystem", International Journal of Electronics and Computer Science Engineering, IJECSE,Volume1,Number 3, 2012

[2] Raghav P. K., Renu Chaudhary, " Compensation of Dispersion in $10 \mathrm{Gbps}$ WDM System by Using Fiber Bragg Grating", IJCEM International Journal of Computational Engineering \& Management, Vol. 15 Issue 5, September 2012

[3] Hani Jasim, " Optical communication system based on wavelength division multiplexing technique", PhD. Thesis, university of Baghdad 2005.

[4] Jackel H.," Optoelectronics and optical communication",2007. http://people.ee.ethz.ch/ fyuriy/oe/oe_optcom chapters/Optoelectronics_2007_Ch10.pdf

[5] Sawsan A. Abdul-Majid and Ahmed A. Abdulla, "using Wavelength-division multiplexing (WDM) to Upgrade nfrastructure Optical Fiber Communication Systems",

[6] Govand P. Agrawal, "Optical Fiber Communication Systems" , $3^{\text {rd }}$ edition, Wiley Interscience, 2002

[7] Gerd Keiser," Optical Fiber Communication ", $2^{\text {nd }}$ edition, Mc Graw-Hill, 1984.

[8] Amin Mohammad. Amin. Dallaali, "Malin Premaratne Power and dispersion constrained optimization of optical links with unequally spaced repeater modules", Optical Fiber Technology, Vol 13, No 4, pp.309-317, October. 2007.

[9] Zou X Y, Hayee M I, H wang S M, et al. Limitations in $10 \mathrm{~Gb} / \mathrm{s}$ WDM optical-fiber transmission when using a variety of fiber types to manage dispersion and nonlinearities[J]. Lightwave Technol., PP: 1144-1152,June,1996.

[10] Bo-ning HU, Wang Jing, Wang Wei and Ruimei Zhao, "Analysis on Dispersion Compensation with DCF based on Optisystem", 2nd International Conference on Industrial and Information Systems, 2010.

[11] Zhaohuaigang, "study on dispersion compensation in optical transmission system", 
study pn optical communications, Vol.3 ,No.141, 2007.

[12] Wangchen,Raomin, "the performance of the DCF Transmissionn system", Journal of applied sciences,Vol.21, No.2,pp.177-181,June 2003.

[13] Killy R I, Thiele H J, Mikhailov v, ea al. Reduction of intrachannel nonlinear distortion in $40-\mathrm{Gb} / \mathrm{s}$ based WDM transmission over standard fiber [J]. IEEE Photonics Technology Letters, 2000, 12(12): 1642-1626.

[14] Eggleton B 1. Dynamic dispersion, compensation devices for high speed transmission systems. Optical Fiber communication conference and exhibit, 2001(3): WH1II-WH1I3.
[15] Djafar K. Mynbaev Lowell L. Scheiner, Fiberoptic communications technology. Beijing: Science publishing company, 2002.

[16] Jianjun Yu, Bojun Yang,"Dispersion-allacated soliton technology with long amplifier spacing and long distance," IEEEphoton technol lett, vol 9, pp. 952-954,No.7, 1997.

[17] Zhou Zhi Qiang, TangYuLiang, "Optimmum schemes of dispersion compensation transmission systems using dispersion compensation fibers",laser technology, VoI.24,No.5,pp.265-269 Oct.2000

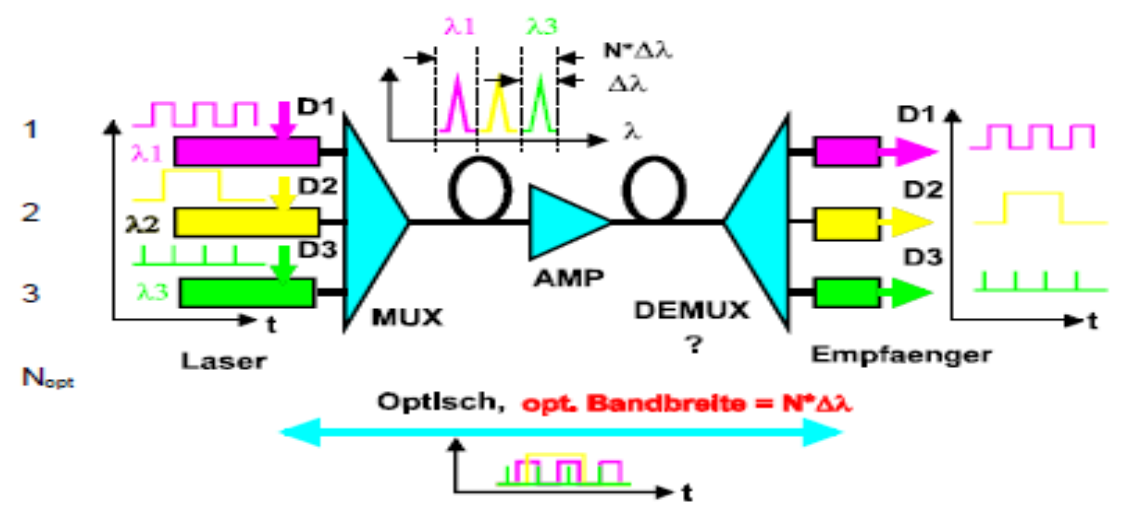

Figure (1): Basic structure of WDM system 


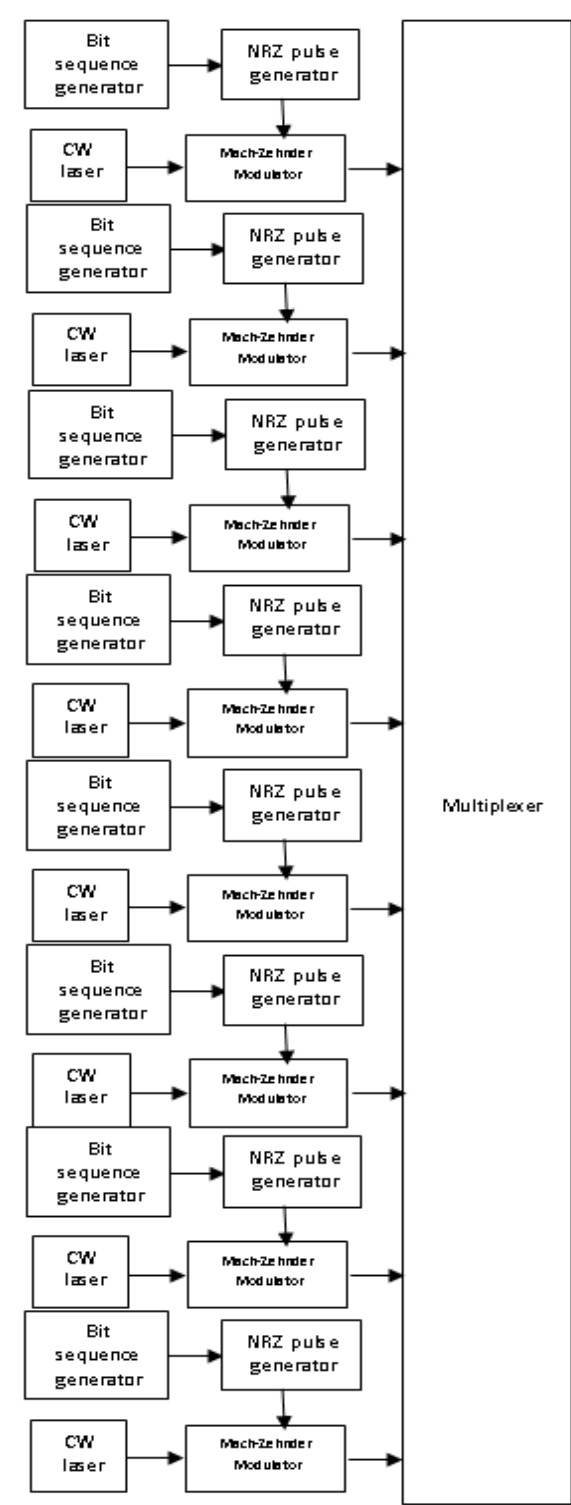

Figure (2): A block diagram of a DWDM system using SMF only.
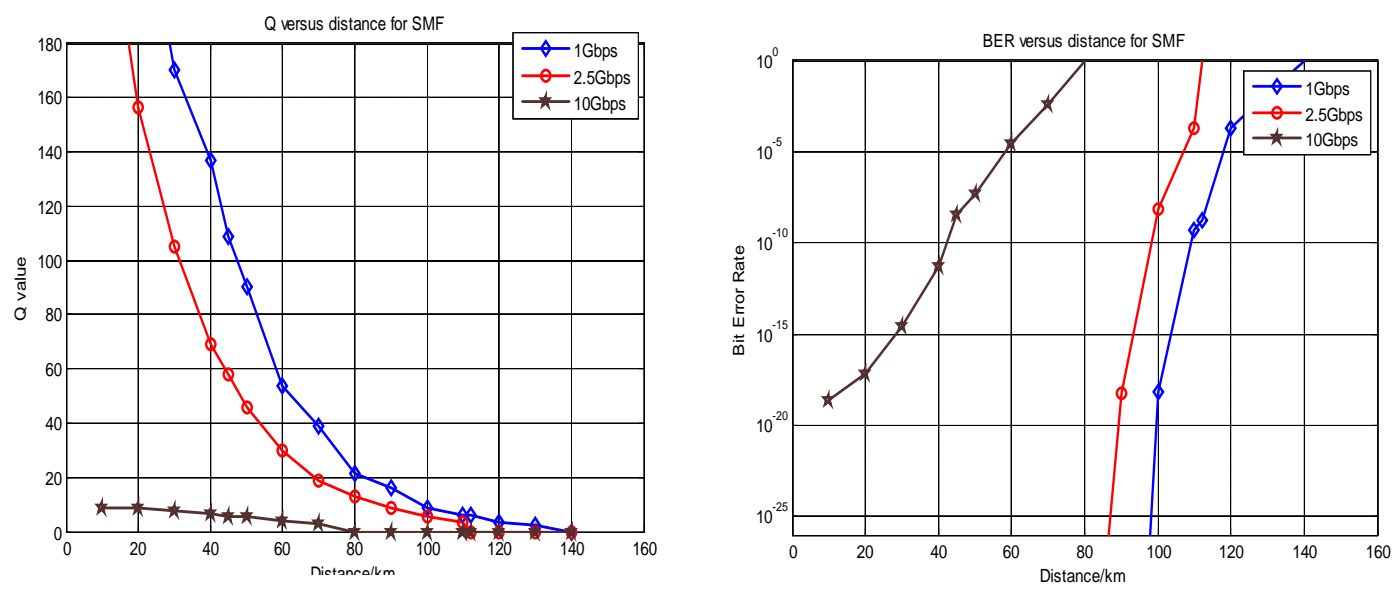

Figure(3): BER and Q factor variation for different lengths of SMF fiber for 1, 2.5 and 10 Gbps bit rates 


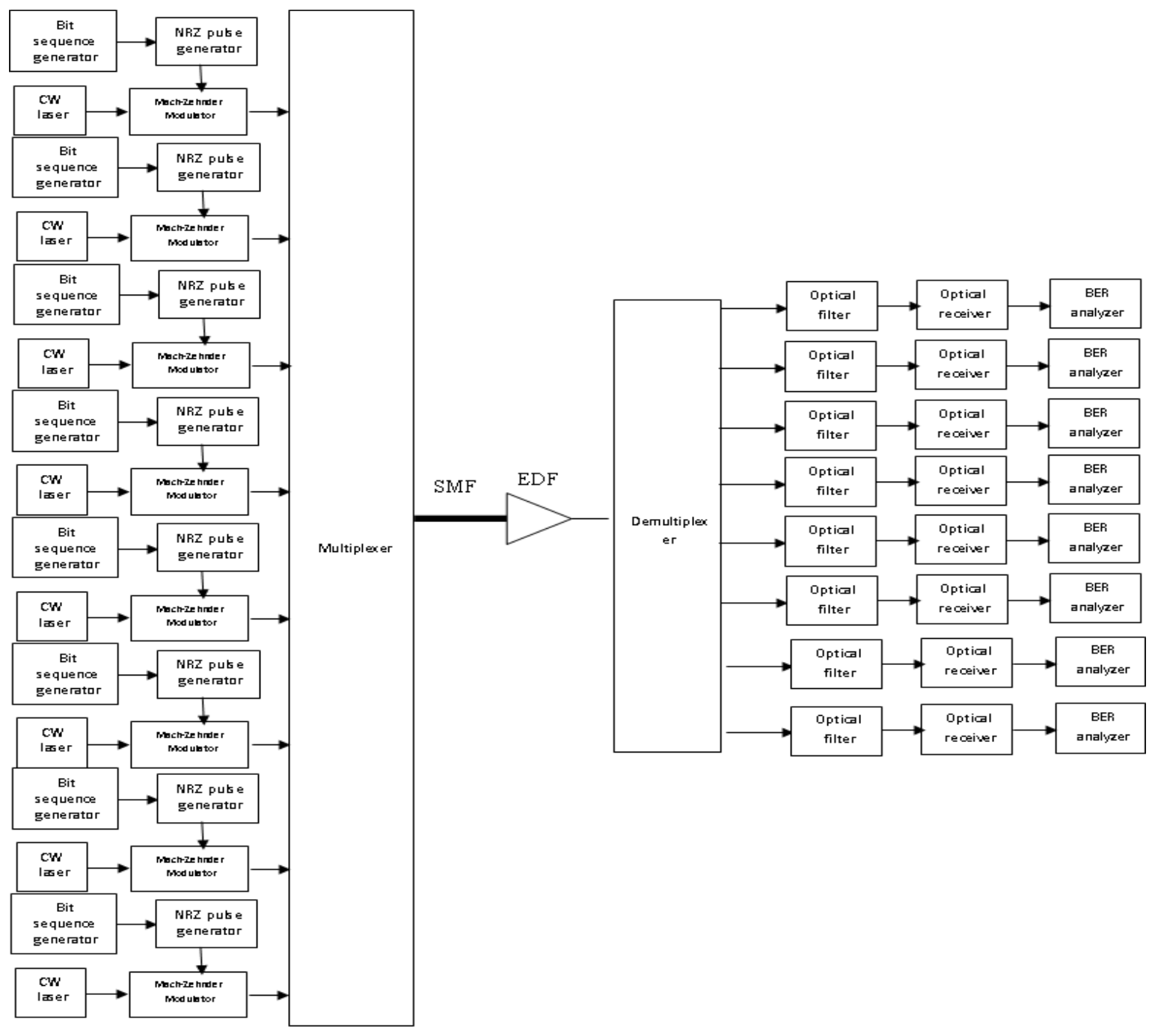

Figure (4): A block diagram of a DWDM system using SMF and EDFA.

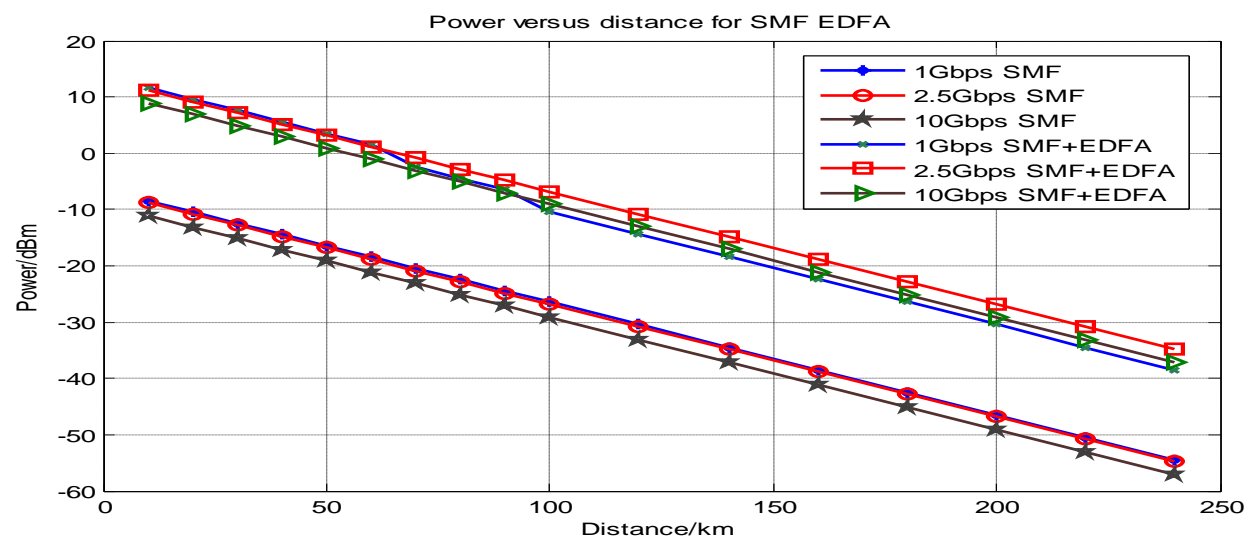

Figure(5): BER and Q factor variation for different lengths of optical fiber of systems using SMF only and SMF and EDFA for 1, 2.5 and $10 \mathrm{Gbps}$ 

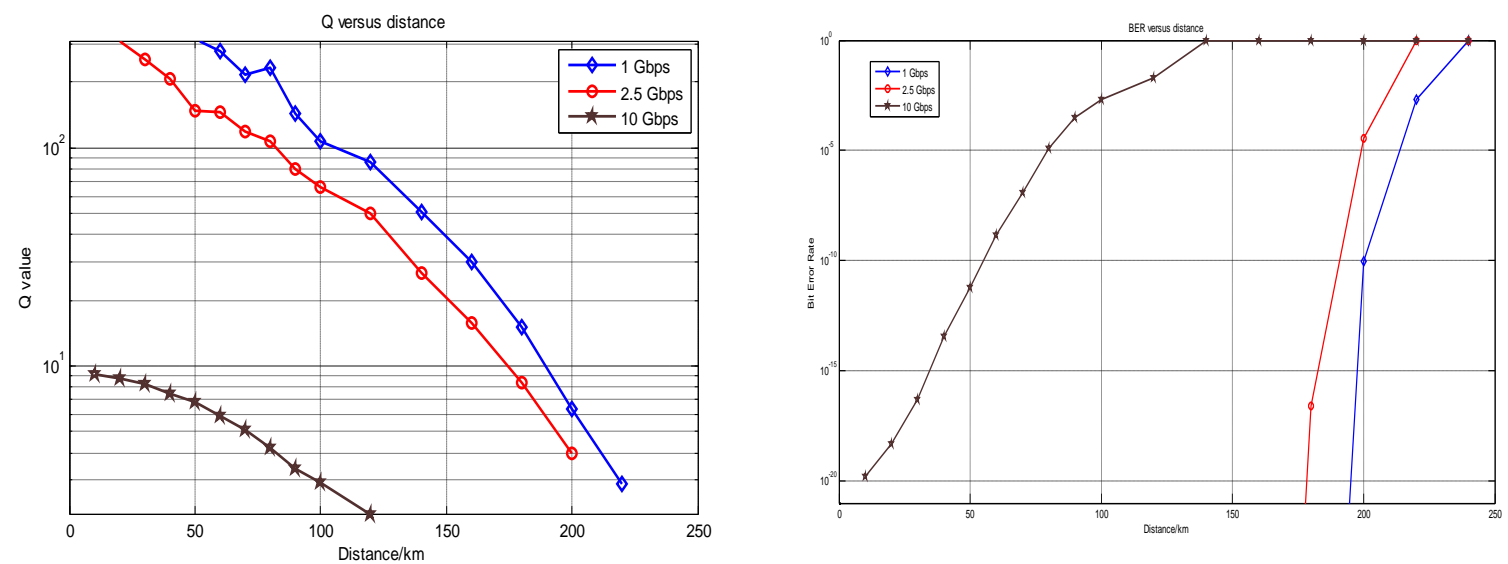

Figure(6): BER and Q factor variation for different lengths of SMF fiber with EDFA amplifier for 1, 2.5 and 10 Gbps.
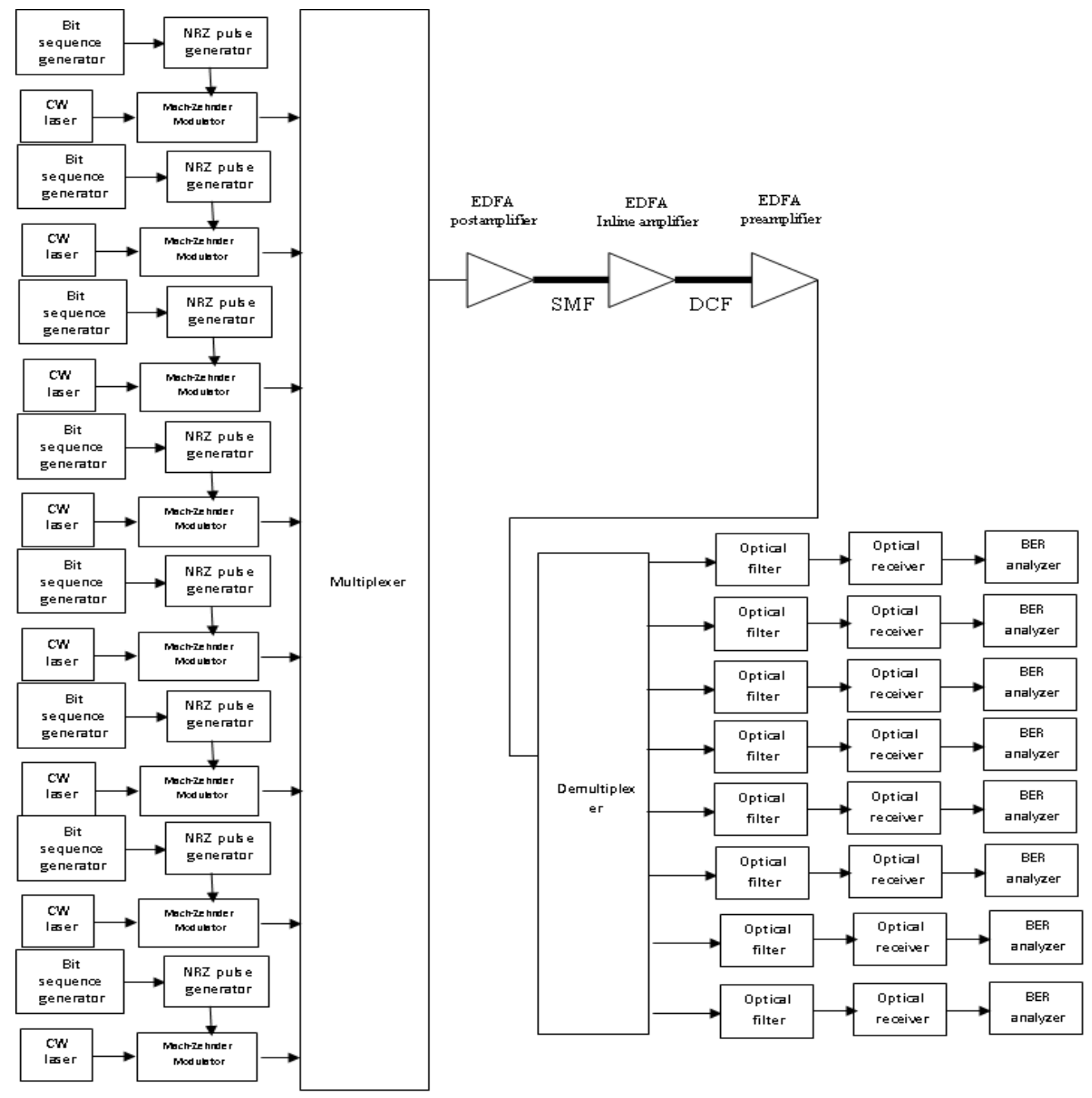

Figure (7): A block diagram of a DWDM system using SMF, DCF and EDFA. 

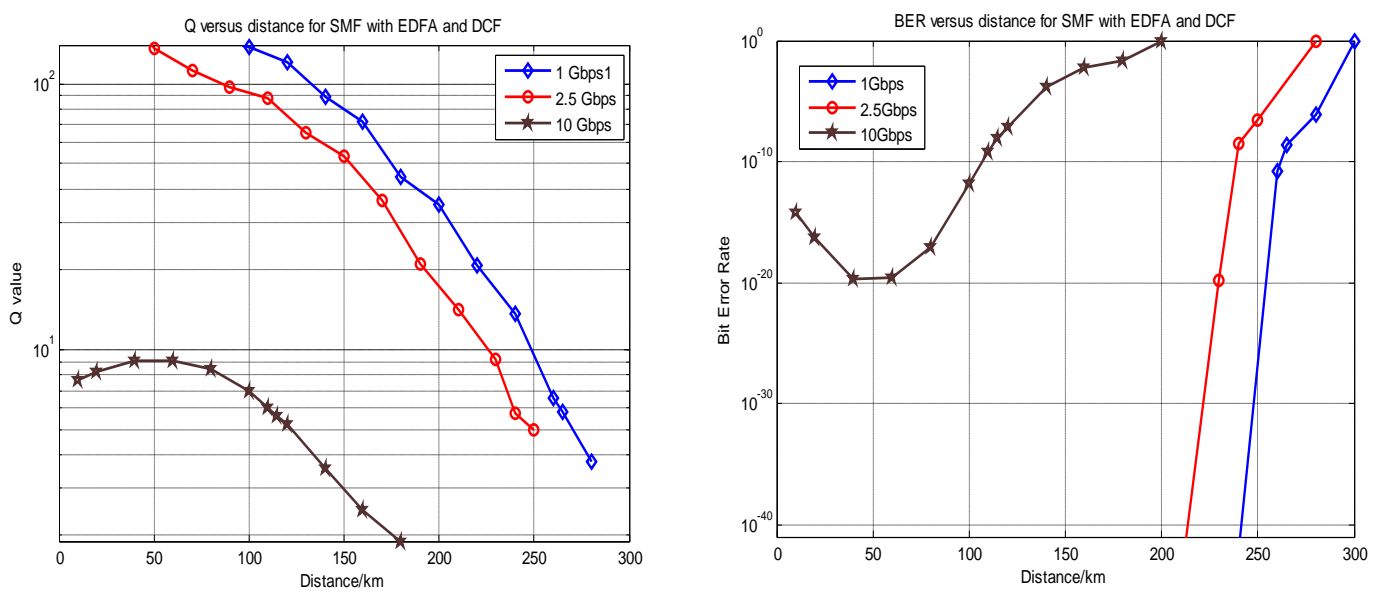

Figure(8): BER and Q factor variation for different lengths of SMF fiber with DCF and EDFA amplifier for 1, 2.5 and $10 \mathrm{Gbps}$
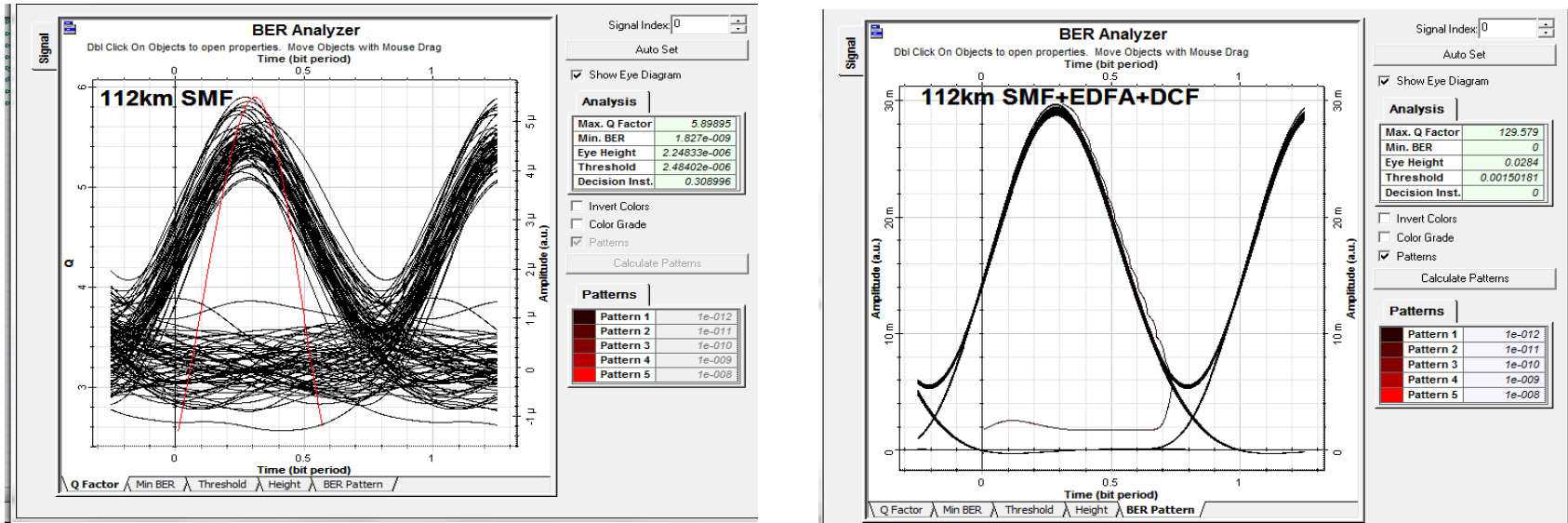

Figure (9): Eye diagram for eight users DWDM with BER of $10^{-9}$ for $1 \mathrm{Gbps}$ bit rate
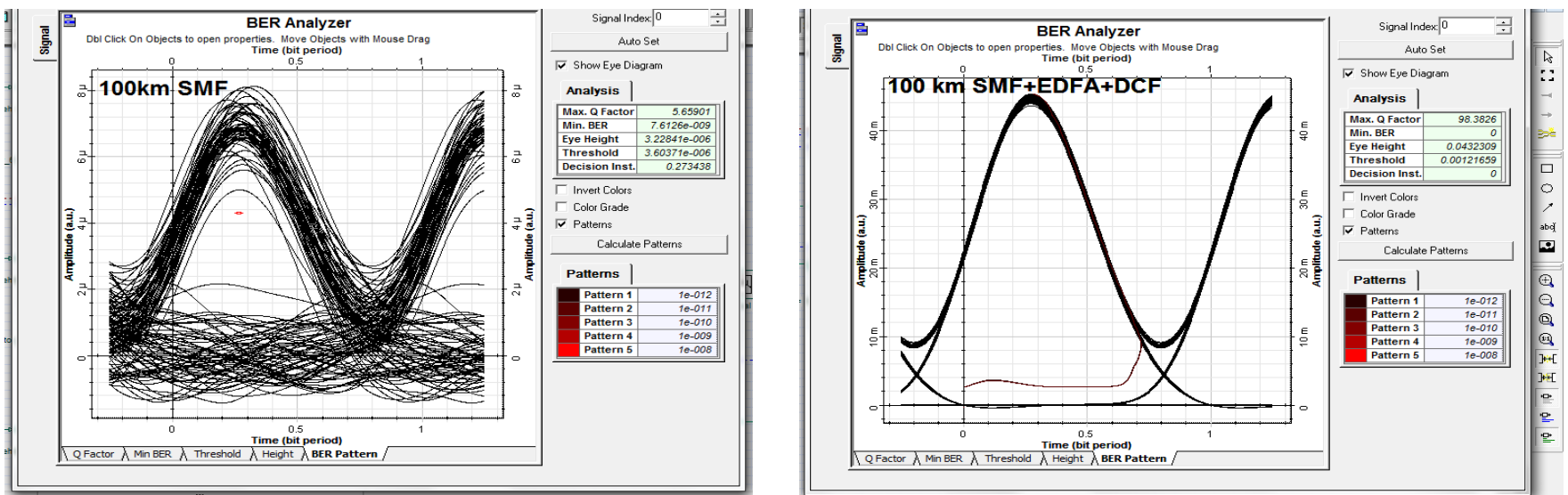

Figure (10): Eye diagram for eight users DWDM with BER of $10^{-9}$ for $2.5 \mathrm{Gbps}$ bit rate 

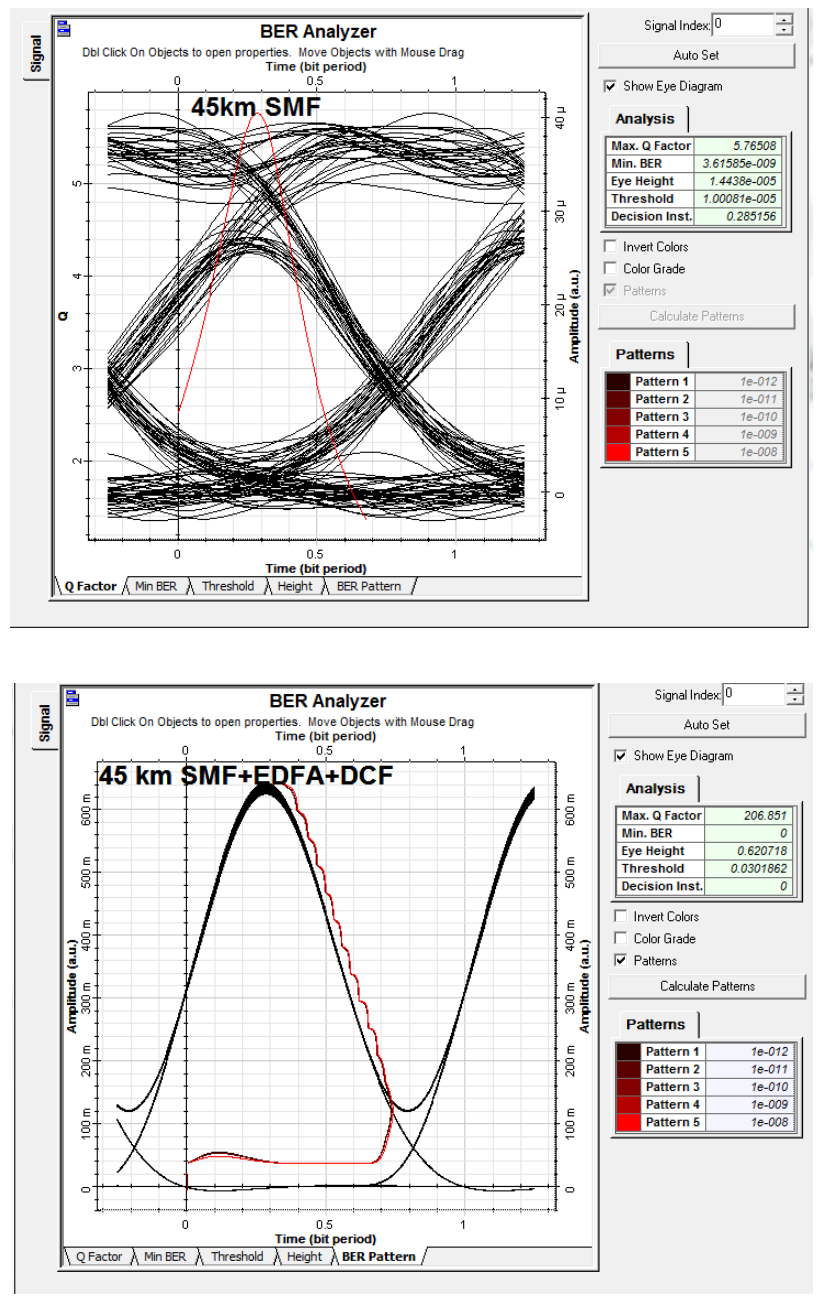
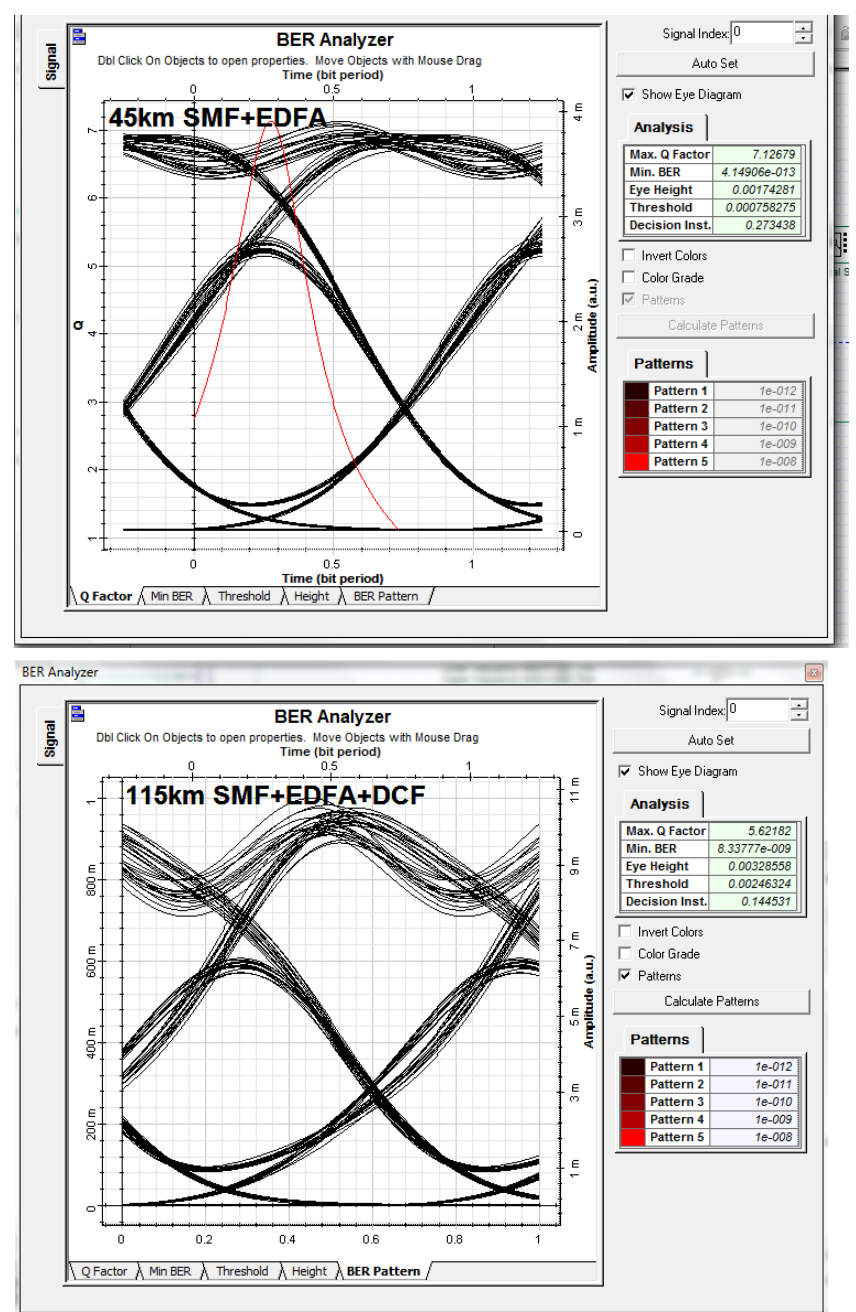

Figure (11): Eye diagram for eight users DWDM with BER of $10^{-9}$ and for 10Gbps bit rate 


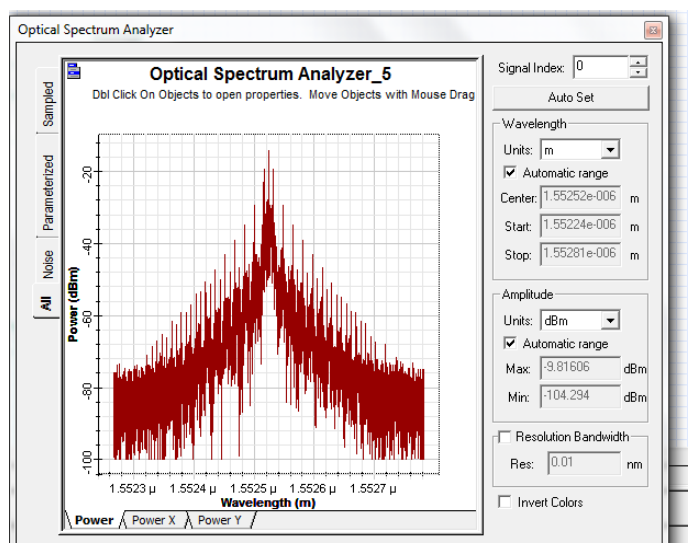

Spectrum at the output of LD

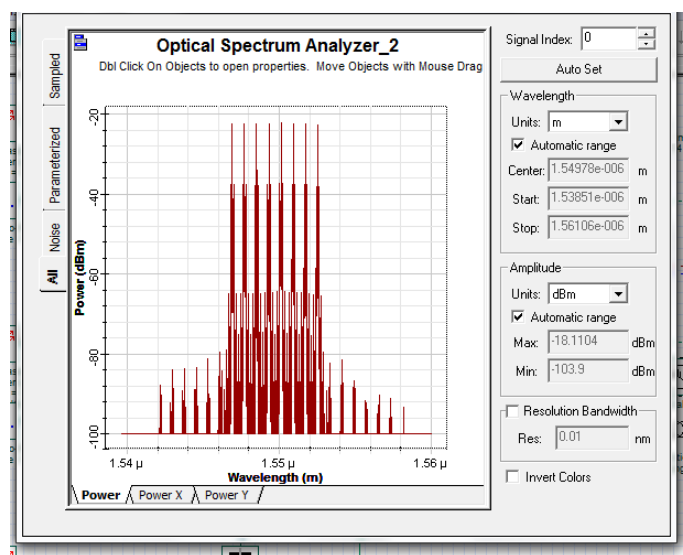

Spectrum after the SMF

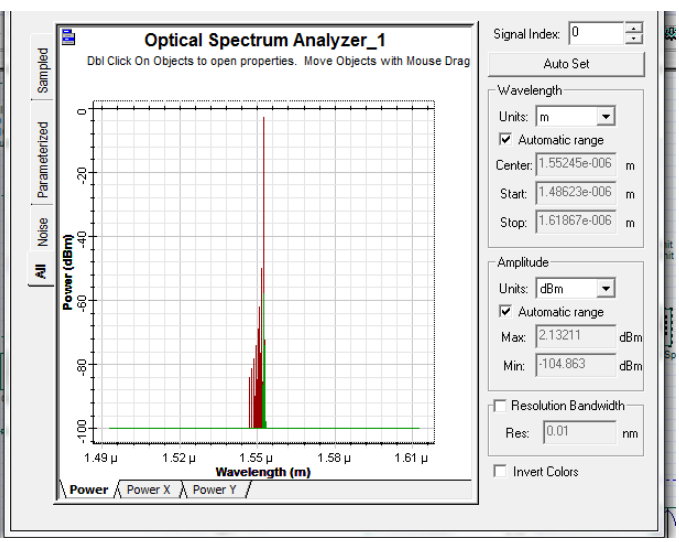

Spectrum after the WDM demultiplexer

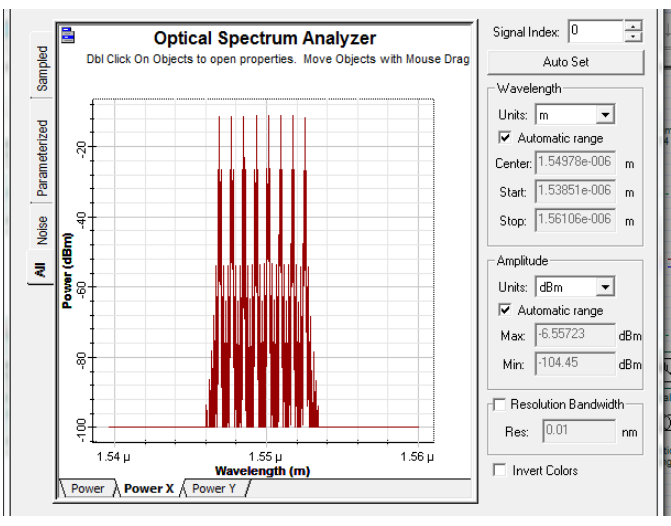

Spectrum after the WDM multiplexer

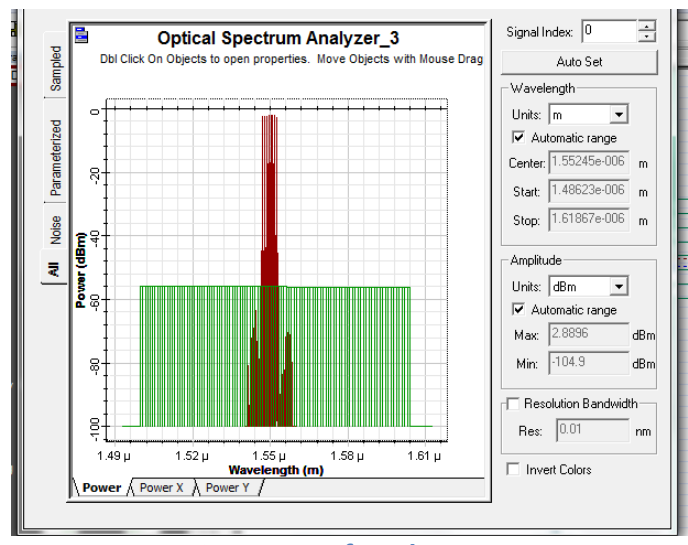

Spectrum after the EDFA

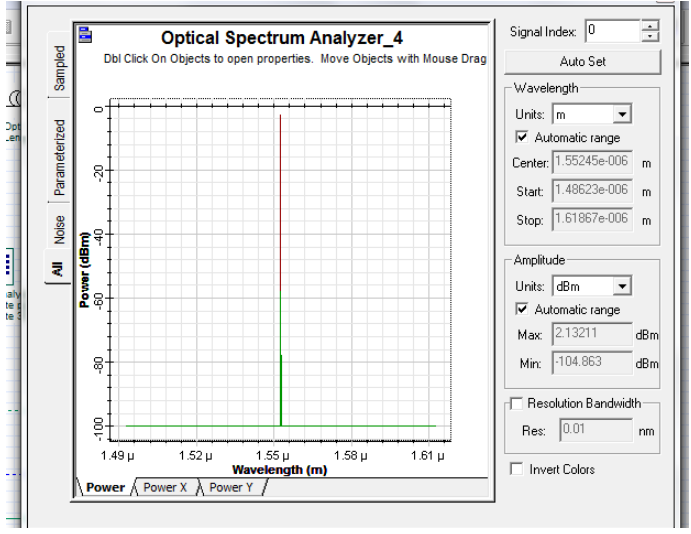

Spectrum after the optical filter

Figure(12): Spectrum of the signal at different points in the system for bit rate of 10Gbps 